\title{
World Psychiatry: trying to reach psychiatrists worldwide
}

\author{
MARIO MAJ
}

Editor, World Psychiatry

My experience as the editor of an international psychiatric journal started very recently: the first issue of "World Psychiatry", the new official journal of the World Psychiatric Association (WPA), appeared in February 2002.

The journal is presently sent free of charge to 20,000 psychiatrists of 114 different countries, whose names and addresses have been provided by the WPA member societies (i.e. the national psychiatric associations of the various countries of the world). An increase of the number of the recipients of the journal up to 30,000 is planned for the near future. At present, in addition to the original edition in English, a Chinese version of the journal is also produced (and sent free of charge by the Chinese Psychiatric Association to all its members). A Spanish, a Portuguese and a Japanese edition are planned for the near future. An electronic version of the journal is also produced and sent by e-mail to the Presidents of all WPA member societies and to the chairpersons of all WPA scientific sections. This electronic version is included in the website of the WPA and of many of its member societies and scientific sections. In the case of some countries, some of the papers of the electronic version appear also in the local language. World Psychiatry does not contain advertisements. A pool of industries have offered to cover the costs of the production and shipment of the journal by an unrestricted grant: they transfer their money

Address for correspondence: Professor M. Maj, Clinica Psichiatrica, Primo Policlinico Universitario, Largo Madonna delle Grazie, 80138 Napoli (Italy).

Fax: +39-081-566.6523

E-mail: majmario@tin.it

Declaration of Interest: no conflict of interest in the connexion with the submitted article. directly to the publisher and are not involved in any phase of the production and distribution of the journal. The publisher of the journal, Masson, has a significant place in the history of the WPA, having been the company which published some of the most important works of the first President and the first Secretary-General of the Association, Jean Delay and Henry Ey.

Mental health care is the main topic of World Psychiatry. Priority is given to contributions dealing with the organization of mental health services throughout the world, to international collaborative research on new models of mental health care, and to reports on innovative experiences in this field. A second priority is psychopathological and biological research which appears solid and relevant to clinical practice. A specific request is made to the contributors to use a very clear and simple language, avoiding concepts and terms which would not be understood by the majority of psychiatrists worldwide. Papers from both developed and developing countries are welcome: among the contributors to the first two issues of the journal, there were three researchers from Africa, five from Asia, six from Latin America and four from Eastern Europe.

The first and most important aim of this initiative is to disseminate information on recent significant clinical, service and research developments, reaching as many psychiatrists of the various countries of the world as possible (including those who have never received their own copy of an international psychiatric journal, as many of the recipients of the journal have told us), using a language that can be assimilated by most of them, focusing on those issues which are relevant to their professional growth and everyday clinical practice, and making an attempt to reach also those psychiatrists who are not able to read English. The second aim is to give voice to psychiatrists from all regions of the world, by encouraging 
submission of research papers, commentaries on topics of interest to psychiatrists worldwide and reports on mental health policy issues or innovative service modalities.

Our main message is that psychiatric research does not need to be complicated and expensive. Ideas are certainly a more important ingredient for psychiatric research than technology and money. Research focusing on mental health services is not less important than biological research, and may be more relevant to clinical practice and more attractive to the majority of clinicians. This kind of research can certainly be carried out also in developing countries. The second message is that we cannot expect that research exerts a significant impact on clinical practice, if clinical practice does not exert a significant impact on the conception and planning of research.

Obviously, World Psychiatry is at present a very imperfect tool to pursue the above aims and to convey the above messages. However, an increasing number of key institutions and leaders from various regions of the world are being involved in the project, including the Department of Mental Health and Substance Dependence of the World Health Organization, and we welcome the support of everyone who is able to contribute ideas.

Is there the need for such an initiative? Are there, at present, problems with the dissemination of information on clinical, service and research developments to psychiatrists worldwide, with the contribution by psychiatrists from non-Western countries to international psychiatric journals, and with the clinical relevance of part of the scientific research which is published in those journals? I think that each of these questions can be answered positively.

The access of the average psychiatrist to international psychiatric journals is becoming more and more difficult. This is not only due to financial reasons (the vast majority of psychiatrists of the world cannot afford a personal subscription to even one international psychiatric journal, and many academic centers are now reducing the number of journals to which they subscribe), but also to the objective difficulty in understanding the language, the concepts and the technical details, and the lack of motivation in perusing articles which do not have an obvious clinical relevance. The traditional gap between the small circle of researchers and the multitude of psychiatric practitioners is thus becoming wider and wider.

That only very few psychiatrists from non-Western countries are able to contribute to international psychiatric journals has been recently documented, and several possible explanations of this situation have been put forward (Patel \& Sumathipala, 2001). Apart from problems with language and research traditions, the fact is that research in psychiatry is becoming an increasingly complex and expensive activity. The contents of our main scientific journals increasingly consist of sophisticated papers reporting on neuroimaging, genetic, or molecular biology research carried out in well-resourced academic departments of a limited number of Western countries. The traditional gap between developed and developing countries in conducting and taking advantage of psychiatric research is thus becoming wider and wider.

The gap between the issues addressed by part of published psychiatric research and those perceived by clinicians as relevant to their everyday clinical practice is probably also increasing. Biological research in psychiatry is crucial for the advancement of knowledge and for the development of new treatment modalities. However, we have been probably too liberal in the last few decades in accepting biological research papers for publication in international psychiatric journals. A look at some of these papers published 10 or 15 years ago may be, in this respect, instructive: the message they conveyed is now completely obsolete, not so much because new technologies have developed in the meantime, but because the findings themselves (usually, a small although statistically significant difference between a sample of patients with a psychiatric diagnosis and a sample of healthy controls, with respect to the mean values for one or more biological variables) were of no actual scientific and clinical relevance. The use of confidence intervals in addition to statistical significance tests, and the provision of information about how many patients and controls presented values above or below one or two standard deviations from the mean for the variable at issue, may be helpful, allowing a clearer evaluation of the scientific and clinical relevance of the findings. Moreover, submitted papers which do not support currently predominant trends and hypotheses should not be penalized with respect to those supporting them: selective publication is not an appropriate way to promote theories, in psychiatry as in any other discipline.

Randomized controlled trials are essential to improve our clinical practice, but the fact that almost all trials published in international psychiatric journals are completely funded by the companies producing the drugs under investigation, and that almost all published trials provide at least some positive results, should raise concern. Moreover, the recent tendency to include in meta-analyses unpublished data deriving from files of drug companies may be misleading. Possible conflicts of interests should be more clearly indicated in papers reporting on drug trials, reports on negative results should be encour- 
aged, and multicenter studies funded by international or national public agencies should be regarded as a priority. We cannot complain that research evidence is not fully transferred to clinical practice, if part of this evidence is regarded by clinicians as biased and non-credible.

Finally, clinical research focusing on the boundaries between various diagnostic categories or on the identification of subtypes within those categories should be given priority for publication only if its practical (e.g. therapeutic) implications are clear.

Psychiatry is still a young discipline, and one in which there are very few certainties. Commercial, academic and other interests are as strong in psychiatry as in other fields of medicine, but the way facts and theories are presented is a more significant factor for their success in psychiatry than in other branches. In this situation, the role of international psychiatric journals is crucial. We should be fully aware of this reality, and do the best we can to confront it.

\section{REFERENCE}

Patel V. \& Sumathipala A. (2001). International representation in psychiatric literature. Survey of six leading journals. British Journal of Psychiatry 170, 406-409. 